https://helda.helsinki.fi

\title{
The quantum epoché
}

\section{Pylkkänen, Paavo}

\section{5}

Pylkkänen, P 2015 , ' The quantum epoché ' , Progress in Biophysics \& Molecular Biology , vol. 119 , no. 3 , pp. 332-340 . https://doi.org/10.1016/j.pbiomolbio.2015.08.014

http://hdl.handle.net/10138/159383

https://doi.org/10.1016/j.pbiomolbio.2015.08.014

other

publishedVersion

Downloaded from Helda, University of Helsinki institutional repository.

This is an electronic reprint of the original article.

This reprint may differ from the original in pagination and typographic detail.

Please cite the original version. 


\title{
The quantum epoché
}

\author{
Paavo Pylkkänen a, b, * \\ a Department of Philosophy, History, Culture and Art Studies \& The Academy of Finland Center of Excellence in the Philosophy of the Social Sciences (TINT), \\ P.O. Box 24, FI-00014 University of Helsinki, Finland \\ ${ }^{\mathrm{b}}$ Department of Cognitive Neuroscience and Philosophy, School of Biosciences, University of Skövde, P.O. Box 408, SE-541 28 Skövde, Sweden
}

\section{A R T I C L E I N F O}

\section{Article history:}

Available online $\mathrm{xxx}$

This article is dedicated to Prof. Basil J. Hiley on the occasion of his 80th birthday.

\begin{abstract}
A B S T R A C T
The theme of phenomenology and quantum physics is here tackled by examining some basic interpretational issues in quantum physics. One key issue in quantum theory from the very beginning has been whether it is possible to provide a quantum ontology of particles in motion in the same way as in classical physics, or whether we are restricted to stay within a more limited view of quantum systems, in terms of complementary but mutually exclusive phenomena. In phenomenological terms we could describe the situation by saying that according to the usual interpretation of quantum theory (especially Niels Bohr's), quantum phenomena require a kind of epoché (i.e. a suspension of assumptions about reality at the quantum level). However, there are other interpretations (especially David Bohm's) that seem to re-establish the possibility of a mind-independent ontology at the quantum level. We will show that even such ontological interpretations contain novel, non-classical features, which require them to give a special role to "phenomena" or "appearances", a role not encountered in classical physics. We will conclude that while ontological interpretations of quantum theory are possible, quantum theory implies the need of a certain kind of epoché even for this type of interpretations. While different from the epoché connected to phenomenological description, the "quantum epoché" nevertheless points to a potentially interesting parallel between phenomenology and quantum philosophy.
\end{abstract}

() 2015 Published by Elsevier Ltd.

\section{Contents}

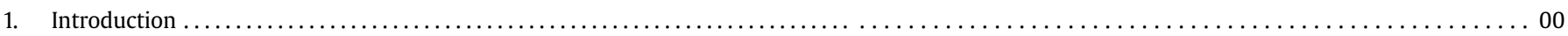

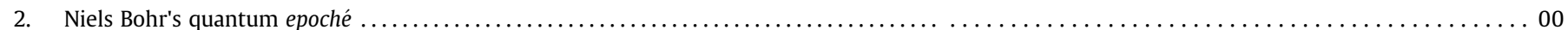

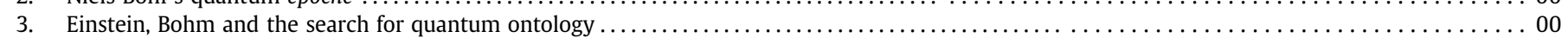

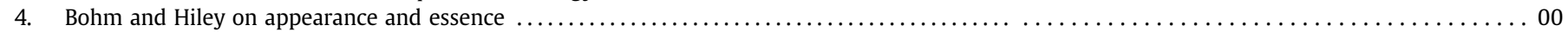

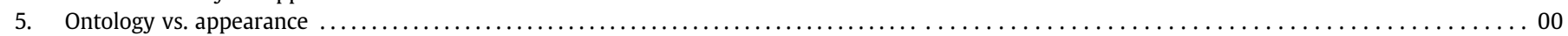

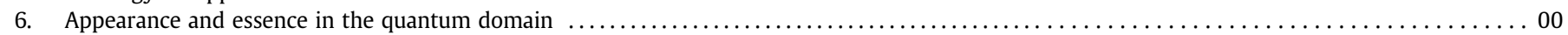

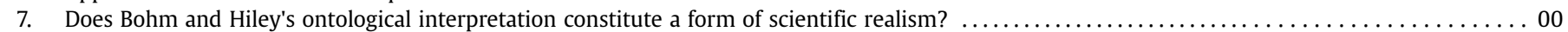

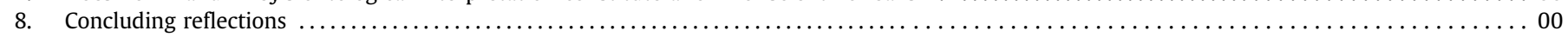

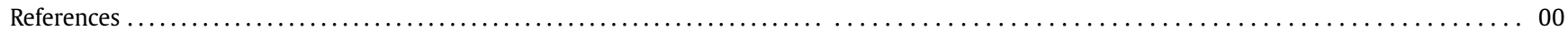

* Theoretical Philosophy, P.O. Box 24, FI-00014 University of Helsinki, Finland. E-mail address: paavo.pylkkanen@helsinki.fi.

\section{Introduction}

There has been relatively little discussion in the phenomenological literature about the radical philosophical implications of quantum theory and relativity. One underlying reason for this may have been Husserl's view of phenomenology as first philosophy, emphasizing that philosophy comes before the special sciences (including physics), in the sense that it studies the preconditions of 
the possibility of our experience of empirical phenomena. While not without insight and value, such a view of first philosophy may make one less prone to see the new philosophical vistas opened up by the empirical sciences, especially physics. Now, this special issue of JPBM as well as the previous research of some of its contributors contain significant attempts to overcome any such oversights. Also, some leading phenomenologists have in fact made connections between phenomenology and the new physics - the most well known example being perhaps Merleau-Ponty's (2003) discussion in his Nature lecture notes (delivered in 1956-1960). One also thinks here about Heidegger's well-known discussions with Heisenberg (see Carson, 2011).

More generally, there were developments in already early 20th century phenomenology that implied the possibility (and indeed the need) to allow the natural sciences to give feedback to the allegedly "first" phenomenological enterprise. For example, Heidegger famously emphasized the role of a "pre-structure" of understanding - the idea being that our experience of meaningfulness is always based on background assumptions and pre-established ways of structuring (Backman 2001: 71). However, Heidegger also emphasized that we are not prisoners of this prestructure. As Backman (2001: 71) describes it:

"The pre-structure is never static ... but lives along with the "hermeneutical circle" of interpretation: in a successful ... process of interpretation the target of interpretation forces us to reconsider the concepts and view from which our interpretation starts from, and grasp them in some way, which in turn influences the experience and interpretation of the target." [translated by PP]

Now, let us assume that our target of interpretation is the new radical empirical results we encounter with quantum and relativistic phenomena, and which physicists first tried to understand in terms of classical physics. In a Heideggerian-hermeneutical way we could suggest that these new phenomena force us to reconsider the classical pre-structure of understanding, to grasp and modify it, and then experience and interpret the quantum-relativistic phenomena in a new, more adequate way. But such modified pre-structure could then be used not only when dealing with novel physical phenomena, but also when trying to describe every-day phenomenal experience in general. A "quantum phenomenology" would then start from first letting the pre-structure of understanding transform and then proceeding to make novel phenomenological descriptions in the light of this new pre-structure. An example of this is David Bohm's (1980) discussion of time consciousness in the light of the notion of implicate order (analysed and connected to Husserl's ideas by Pylkkänen, 2007).

It is typical of later phenomenology, especially Heidegger that there is much emphasis on the historical situation one finds oneself in, as well as on the situation and context in which meaningful phenomena unfold (see e.g. Westerlund, 2014). This is another area where connections to quantum and relativistic phenomena seem relevant. Regarding historicity, Heidegger emphasized that "philosophy" is a limited historical phase of Western thinking. As Backman (2001: 76) describes it, "philosophy" in this Heideggerian sense is metaphysical thinking aiming toward absolute generality that begins with Plato and Aristotle, but which has come to an end especially as a result of the thought of Hegel, Marx and Nietzsche. However, quantum and relativistic phenomena may give a new challenge and a possibility for renewal (rather than end) even for "philosophy" in this traditional sense. And when it comes to situationality and contextuality, there are some interesting analogues to phenomenological themes especially in some interpretations of quantum theory, as we will see below.
There are thus a number of reasons why bringing together phenomenology and quantum-relativistic physics is potentially fruitful. For one thing this might enable new, more insightful phenomenological descriptions, as Bohm's above-mentioned discussion of time consciousness illustrates. Also, the methodological insights of phenomenology might prove useful when trying to make sense of the interpretational puzzles of quantum theory in particular (for example, we will below consider how a kind of epoché or suspension of assumptions about reality at the quantum level seems to be required to make coherent sense of the results of some quantum mechanical experiments).

Further, such bringing together of phenomenology and the new physics also seems to be in line with the very criteria phenomenologists typically set to themselves. The empirical phenomena encountered in quantum and relativity physics have given rise to a new philosophical situation that poses a challenge to not only "philosophy" in a Heideggerian sense, but also to phenomenology broadly understood. Given the emphasis that (later) phenomenology puts upon historicity and situationality, it is somewhat ironical that there have been so few phenomenological discussions of the new epistemic and ontological situation opened up by quantum theory and relativity. However, this also implies the possibility for much fruitful research in the future. For example, there has been an extensive philosophical discussion of the meaning of quantum theory since the 1920s. Connecting this rich discussion with relevant aspects of phenomenological thought suggests a vast research programme, with possibilities of mutual criticism and enrichment between the two approaches. For examples of such attempts see Honner (1987), and (especially in relation to "post-phenomenology”) Plotnitsky (1994), Globus (2003) and Pylkkö (1998). And of course, as already mentioned, this special issue of JPBM, as well as the previous research of some of its contributors, marks significant progress to a new synthesis of phenomenology, physics and biology (see also Globus et al., 2004).

In this article the theme of phenomenology and quantum physics is tackled by examining some basic interpretational issues in quantum physics. One key issue in quantum theory from the very beginning has been whether it is possible to provide a quantum ontology of particles in motion in the same way as in classical physics, or whether we are restricted to stay within a more limited view of quantum systems, in terms of complementary but mutually exclusive phenomena. In phenomenological terms we could describe the situation by saying that according to the usual interpretation of quantum theory (especially that due to Niels Bohr), quantum phenomena require a kind of epoché (i.e. a suspension of assumptions about reality at the quantum level; cf. Hut, 2001). However, there are other interpretations (especially that due to David Bohm) that seem to re-establish the possibility of a mindindependent ontology at the quantum level. But as we will see, even such ontological interpretations contain novel, non-classical features, which require them to give a special role to "phenomena" or "appearances", a role not encountered in classical physics. We will conclude that while ontological interpretations of quantum theory are indeed possible, quantum theory implies the need of a certain kind of epoché even for this type of interpretations. While different from the epoché connected to phenomenological description, the "quantum epoché" nevertheless points to a potentially interesting parallel between phenomenology and quantum philosophy.

Our discussion also connects to some of the central issues discussed by Kauffman and Gare (2015) in their Prologue to this special issue of JPBM. For one thing, there is the question of measurement in quantum mechanics. Kauffman and Gare explore the possible role of "mind" in quantum measurements. They present the "Triad", a new view of quantum mechanics which has 
"Actuals that are ontologically real and defined as obeying Aristotle's law of the excluded middle, Possibles which are ontologically real and are defined as not obeying the law of the excluded middle, and Mind, consciousness with qualia, and free will doings at measurement. In short: res potentia, res extensa linked by "mind" at measurement." (Kauffman and Gare, 2015).

Now, Bohm showed in 1952 that measurement-like processes at the quantum level can take place objectively, without any active role of human consciousness (for a good introduction to how the Bohm theory solves the measurement problem, see Ney, 2013: 26-32; for a more extensive presentation, see Bohm and Hiley, 1993; ch 6; see also Hiley and Pylkkänen, 2005). In Bohm's quantum ontology an electron is assumed to be a particle guided by a new type of quantum field (which latter is mathematically described by the Schrödinger wave function). In Kauffman and Gare's terms, Bohm showed that res potentia (understood as a quantum potential which arises from the quantum field) acts on res extensa (the localized particle aspect of an electron) in such a way that we get definite results of measurement, without Schrödinger's cats or many worlds. And all of this can happen objectively, without the need for human observers or minds. According to the Bohm theory measurement processes are a special case of quantum processes (such as transitions between stationary states) which latter take place in nature completely independently of human intervention (Bohm and Hiley, 1993: 97).

However, a deeper study of the Bohm theory suggests an affinity with Kauffman and Gare's approach. Note especially that Bohm and Hiley $(1987,1993)$ suggested that the quantum field is not pushing and pulling the particle mechanically, but is rather in-forming the energy of the particle. Bohm (1990) characterized the quantum field as containing "active information" and saw this as a primitive mind-like property of elementary particles such as electrons. This is in some ways similar to Kauffman and Gare's ideas about some kind of "proto-consciousness" being present in quantum processes.

There is also a connection to the issue of exophysics (which presupposes a detached observer) and endophysics (which sees the observer and measurement as part of the domain that needs to be accounted for; for details see Kauffman and Gare's Prologue). First of all, there is a sense in which the Bohm theory allows us to retain an exophysical perspective to the universe. For if we assume that human observers have no significant influence upon the quantum state of the universe, then we can for all practical purposes ignore measurements and the active role of human observers in our general description of nature (remembering, however that Kantian considerations suggest that we probably have no perceptual and conceptual access to nature from a non-human perspective; see Pylkkänen, 2015a). However, there is also a sense in which the Bohm theory fits very well together with endophysics. For one thing, the Bohm theory provides perhaps the clearest available account of how the process of measurement can be accounted in terms of quantum theory itself, thus enabling a coherent endophysical discussion of quantum measurements.

Bohm also sketched how the consciousness of the observer could be included into the picture. The basic idea is that the quantum ontology needs to be extended. At the quantum level we have the particles guided by the quantum field of information. Bohm speculated that in the context of complex biological systems such as brains there is likely to be a whole hierarchy of such fields of information, ranging from "manifest" to more "subtle", and actively enfolding and unfolding information about each other, involving guidance relations. Mental processes and conscious experience could then come in at the more subtle levels of organization. Through the hierarchy of fields of information, mental processes and perhaps a kind of "free will" could make a difference to more manifest physical processes via the quantum field, suggesting another affinity to the ideas of Kauffman and Gare (as well as to those of Penrose of Hameroff; see Bohm (1990: 283); Pylkkänen, 2015b). Further, in his more general implicate order scheme Bohm sketched how both biological and mental processes could be understood in relation to the physical world, as described by quantum and relativity physics (see Bohm, 1980; Pylkkänen, 2007).

The above considerations are meant to indicate that there are rich connections between Bohm and Hiley's natural philosophy to the general approach discussed in this special issue, especially as it is excellently brought out by Kauffman and Gare. In the rest of this article we will, however, focus on the question about the role of phenomena or appearance in Bohr's and Bohm's interpretations of quantum theory.

\section{Niels Bohr's quantum epoché}

Can we have an ontological description of quantum systems say, of particles such as electrons? In the domain of classical physics it is possible to measure simultaneously the position and momentum of a particle with arbitrarily good accuracy within a single phenomenon, and thus one is allowed to postulate a particle ontology, in other words to make the transition from phenomena to ontology. Usually in classical physics one just talks about the particle and its properties, ignoring the phenomenon in which these were measured (Bohm and Hiley, 1993). In phenomenological terms, this reminds us of the "natural attitude" - we take for granted that the objects that appear to us in experience also exist when not being observed, we slip from appearance to reality without noticing it.

However, according to Niels Bohr (1949) we cannot make a separation between the particle and the experimental arrangement in quantum situations, because the quantum of action that connects them during a measurement is indivisible, unpredictable and uncontrollable. For Bohr this meant that we cannot analyse the quantum phenomenon and assume that we are getting information about a separately existing particle with well-defined properties. Note in particular that there is no single phenomenon in which, say, both the position and the momentum of a quantum system can be accurately measured at the same time. Thus, implied Bohr, it is not legitimate or meaningful to postulate an ontology in which a particle has simultaneously a well-defined position and momentum, as these supposedly exists irrespective of any act of observation. In phenomenological terms we could say that Bohr was calling for a kind of epoché in the context of quantum phenomena, in the sense that we need to suspend our classical habit of conceiving of a welldefined, observation-independent ontology of particles in motion.

So the issue for Bohr seems to be when it is legitimate to postulate an ontology. Or, to put it differently, what does the incompatibility of phenomena associated with a single system imply for the ontology of that system? The following quote captures many essential features of Bohr's (1949) subtle ideas:

"This crucial point ... implies the impossibility of any sharp separation between the behaviour of atomic objects and the interaction with the measuring instruments which serve to define the conditions under which the phenomena appear. In fact, the individuality of the typical quantum effects finds its proper expression in the circumstance that any attempt of subdividing the phenomena will demand a change in the experimental arrangement introducing new possibilities of interaction between objects and measuring instruments which in principle cannot be controlled. Consequently, evidence obtained under different experimental conditions cannot be 
comprehended within a single picture, but must be regarded as complementary in the sense that only the totality of the phenomena exhausts the possible information about the objects." (Bohr in Schilpp, 1949).

The phenomenological reader notices how the measuring instruments play a "transcendental" role for Bohr, as they define the very conditions under which the phenomena appear (cf. Honner, 1987; Husserl, 1913/1976). A new kind of wholeness in physics is implied by the fact that no sharp separation between the behaviour of atomic objects and the interaction with the measuring instruments can be made. This means that we typically cannot assume, when looking at the result of measurement in an experiment, that the result refers to the behaviour of the atomic object as it unfolds independently of its being measured. This is in stark contrast to the situation in classical physics, where the theory allows us to calculate the influence of the measuring instruments to a good approximation and in this way get as accurate information about the behaviour of the object as we want.

Note also how Bohr says that it is because of such indivisibility or "individuality" that different experimental arrangements are mutually exclusive. If I want to measure the momentum of a particle in a situation where I am measuring its position, I need to make changes in the experimental arrangement. However, such changes introduce new possibilities of interaction between objects and measuring instruments which, Bohr says, in principle cannot be controlled. This connects with the uncertainty principle and Bohr's assumption that the quantum of action needed in each measurement is uncontrollable. After the changes in the experimental arrangement required to measure the momentum of the particle I can no longer measure its position. Thus the experimental arrangements are mutually exclusive. Bohr further emphasizes that we cannot comprehend the evidence from such mutually exclusive arrangements within a single picture. And yet, we have different kinds of evidence about the same object. How should we consider such evidence concerning the same object, evidence that is obtained from mutually exclusive, incompatible experimental phenomena? Bohr says that we must consider such evidence as complementary. Notice that "complementary" here cannot be understood in the sense of two parts of a single picture. Rather, we have here two incompatible pictures which, however, both give information about the object. Bohr says that “... only the totality of the phenomena exhausts the possible information about the objects". But this totality includes mutually exclusive phenomena. Bohr's notion of complementarity is thus very subtle, involving the necessity to combine incompatible viewpoints. Arkady Plotnitsky (2010: xvi) provides a succinct summary of this difficult concept:

“... complementarity is defined by (a) a mutual exclusivity of certain phenomena, entities, or conceptions; and yet (b) the possibility of applying each one of them separately at any given point; and (c) the necessity of using all of them at different moments for a comprehensive account of the totality of phenomena that we must consider."

As we have noted above, complementarity also implies that we cannot analyse the quantum phenomenon and assume that we are getting information about a separately existing particle with welldefined properties. Note however that in recent years there has been an increasing interest in so-called "weak measurements" in quantum mechanics. While even these can be understood in terms of Bohr's complementarity, they nevertheless open up the possibility for new ways of thinking about quantum measurement (see Flack and Hiley, 2015, and the references therein).
Bohm and Hiley (1993: 16) describe the unanalyzable and holistic aspects of Bohr's view succinctly:

"... the quantum link connecting the experimental result with its meaning is indivisible, unpredictable and uncontrollable. The meaning of such a result can, therefore, no longer be coherently described as referring unambiguously to the properties of a particle that exists independently of the rest of the phenomenon. Instead this meaning has to be regarded as an inseparable feature of the entire phenomenon itself. Or, to put it more succinctly, the form of the experimental conditions and the content (meaning) of the experimental results are a whole, not further analysable. It is this whole that, according to Bohr, constitutes the quantum phenomenon."

Notice that, according to Bohm and Hiley, there is a relationship between form and content in a new, special sense in Bohr's view. The form of the experimental conditions and the content or meaning of the experimental results are assumed to be an unanalyzable whole. This means that it is not possible to isolate a particle from this phenomenon, and think about it in the traditional realist fashion.

Bohr's holistic view implies limits to the visualisation of atomic phenomena - for example, he thought that it is not possible to provide trajectories for quantum particles. He underlined that the "renunciation of the visualisation of atomic phenomena is imposed upon us by the impossibility of their subdivision" and referred to "the impossibility of subdividing quantum phenomena and ... the ambiguity in ascribing customary physical attributes to atomic objects". There is an

“... essential ambiguity involved in a reference to physical attributes of objects when dealing with phenomena where no sharp distinction can be made between the behaviour of the objects themselves and their interaction with the measuring instruments" (1949).

Finally, Bohr did not think that the situation encountered with quantum phenomena was just a temporary state of affairs, to be overcome in future developments:

“... in quantum mechanics, we are not dealing with an arbitrary renunciation of a more detailed analysis of atomic phenomena, but with a recognition that such an analysis is in principle excluded." (1949).

Bohr's claim about the need for a renunciation of the visualisation of atomic phenomena is interesting from a phenomenological point of view. Note in particular that the late Heidegger's post-metaphysical thought emphasized that the appearance of a certain content is made possible only in relation to a background context which itself does not appear (Backman, 2010: 77). Somewhat analogously, Bohr's complementarity implies that a given aspect (e.g. position) can fully appear only if we give up the attempt to make a complementary aspect (e.g. momentum) appear. In terms of David Bohm's later implicate order approach (which is in some ways similar to Bohr's view, but differs in that it is more ontological and general) we could say that not every relevant property of a particle can be made explicate or manifest at the same time - every quantum particle, and thus the entire universe lives in an "intrinsically implicate order" which can unfold creatively to constitute the present moment (Bohm, 1980; Pylkkänen, 2007). For example, if a given variable (e.g. position) is explicated in an experiment, its complementary variable (momentum) is left "implicate" and vice versa. Thus Bohm's implicate order scheme, like the late Heidegger, 
emphasizes that for something to appear there must be an entire implicate background which cannot be made to appear at the same time. And Bohr assumed that it is not possible to go consistently beyond this phenomenologically limited situation, by visualizing quantum systems with all their properties present simultaneously, as one does in classical physics. As we will see, it was Bohm who made such "impossible" visualization possible in his 1952 theory. Ironically, there is a sense in which Bohm's 1952 scheme with its more explicate ontology is in opposition to the more general implicate order scheme he developed since the 1960s (for Bohm's own attempt to bring the 1952 approach and the implicate order approach together, see Bohm, 1987).

\section{Einstein, Bohm and the search for quantum ontology}

For Albert Einstein the programmatic aim of physics was a complete description of any individual real situation as it supposedly exists irrespective of any act of observation (1949). In other words he felt that physics ought to strive for a kind of "exophysical" description that Bohr thought was in principle impossible. There was thus, as is well known, a fundamental disagreement between Bohr and Einstein about the interpretation of quantum theory.

As we have already mentioned many times above, a very important contribution to this debate was made by David Bohm. After several "interesting and stimulating" discussions with Einstein in Princeton, Bohm published in 1952 an interpretation of quantum theory in terms of "hidden variables". He realized that Schrödinger's equation implies a well-defined, visualizable, unambiguous ontology where an electron is a particle always accompanied and guided by a new type of quantum field, described by the wave function. He had independently rediscovered and improved the pilot-wave theory proposed by de Broglie already in the 1920s (for an appraisal of de Broglie's contributions see Bacciagaluppi and Valentini, 2009; for a critical perspective on their discussion see Holland, 2011).

Bohm's theory was not, however, a return to classical physics. Although his 1952 theory was deterministic, already in 1954 he published with Vigier a stochastic version of the theory, thus underlining that the key point of the theory was the possibility of a quantum ontology, not the issue of determinism (Bohm and Vigier, 1954; see also Bohm and Hiley, 1993, ch 9). Also, as Bohm and Hiley brought out more clearly later (1975), the Bohm theory was nonlocal and suggested the need for a new holistic description of quantum system. Now, we already saw above that a certain kind of quantum wholeness was a key aspect of Niels Bohr's interpretation of quantum theory. So while there is a sense in which the Bohm theory differs from Bohr (e.g. in enabling us to analyse or "break" Bohr's indivisible quantum phenomenon conceptually in terms of a particle guided by a quantum field), there are also important similarities.

Bohm's interpretation was initially resisted, but is today more and more widely acknowledged as one of the key possible interpretations of quantum theory. Later on further ontological models were proposed, for example Everett's 1957 "many worlds" interpretation and Ghirardi, Rimini and Weber's 1986 objective collapse theory, and currently the nature of quantum reality is intensively debated within the philosophy of physics community (see e.g. Saunders et al. eds. 2010; Ney and Albert eds. 2013). We do not know which ontological interpretation (if any) is correct, but each may reveal something significant about the nature of physical reality at a very fundamental level. One should note that there are by now also different versions of the Bohm theory. Much attention has in recent years been given to a minimalist version known as "Bohmian mechanics" (see e.g. Goldstein, 2013). Bohm himself developed since the mid-1970s, with Basil Hiley, a philosophically more radical version they called the "ontological interpretation", culminating in their 1993 book The Undivided Universe. We will here be concerned with Bohm and Hiley's later development of the Bohm theory (for an insightful and entertaining presentation of the Bohm theory, see Towler, 2009; see also Pylkkänen et al., 2015).

In particular, we will consider what the Bohm theory can offer in place of Bohr's "quantum phenomenon". The key point is that in Bohm's quantum ontology, too, the object and the apparatus participate irreducibly during a measurement. This means that the phenomena that reveal themselves in the apparatus (i.e. the results of measurement) typically cannot be referred to the object alone, but only to the totality of apparatus and object. This is very reminiscent of Bohr's view, but with the difference that we can now understand the way in which this peculiar, irreducible role of the quantum phenomenon is a result of the holistic features of the quantum reality of particles and field.

Bohm and Hiley also explain their philosophical position in some detail in The Undivided Universe. Although they are emphasizing the possibility of a consistent quantum ontology, it is clear that they are not advocating any simplistic form of scientific realism. For example, when they explain their general world-view and consider epistemic issues, they say: "ultimately all of our thought [including theories] can be regarded as appearance, not to the senses, but to the mind" (1993: 322). Now, does this mean that theories, in the end have to do with the way the world appears to be rather than the way the world is? This sounds like a concession to Bohr's approach that emphasizes that we cannot go beyond phenomena when dealing with atomic objects.

Traditionally ontology refers to the way the world is. A brief etymological detour gives us some useful terminology. According to one interpretation, the word "ontology" comes from the Greek logos peri ta onta, where ta onta means "the beings", logos has meanings like "word", "speech", "reason", and peri means "around", and perhaps in this context "about". So "ontology" originally would have meant something like "reasoning about the beings" (Sampo Vesterinen, private communication). So, in these terms, are Bohm and Hiley reasoning about the beings or about the way the beings appear? Or do they suggest that everything is, in the end appearance?

There is thus at least prima facie a creative tension between the claim that their theory gives us an "ontology" and the claim that theories constitute "appearances". As already mentioned, this tension is particularly interesting when considering the relation of their view to that of Bohr. One might think that the whole point of an ontological interpretation of quantum theory is to try to go beyond Bohr's "quantum phenomenon". Indeed, this is what Bohm and Hiley (1993: 25-6) set out to do. But as we will see, in the end their quantum ontology suggests that the relation between "appearance" and the underlying reality or "essence" in a quantum situation is radically different from that in classical physics. Their view has some striking similarities to Bohr's view, while there are significant differences as well.

\section{Bohm and Hiley on appearance and essence}

To understand Bohm and Hiley's (hereafter $\mathrm{BH}$ ) views about "appearance" and "essence" in the quantum domain, as described in The Undivided Universe, it is useful to first consider their view about scientific theories in general. They propose that there is no final theory. This implies limitations to what we can say about the universe as a whole on the basis of a scientific theory. For example, we cannot decide on the basis of a particular theory, such as quantum theory, whether the universe is ultimately deterministic or indeterministic. And thus we cannot use physical theories to draw conclusions about, say, the ultimate limits of human freedom 
(BH 1993: 3).

Their view here resembles that of Kant, who held that physical theories only apply to the phenomenal world, while the thing-initself remains unknown. Thus any determinism that physical theories may involve does not limit human freedom insofar as this is grounded beyond the phenomenal world. Colin Marshall (2012) describes Kant's view succinctly:

"Kant holds that much of reality remains unknowable, yet he takes this to be a desirable result, for it precludes scientific considerations (which only concern the appearance-based properties of objects) from ever ruling out freedom of the will

Although BH's emphasis on ontology may suggest to some that they are engaged with pre-Kantian (Aristotelian) metaphysics, we will see that the issue is more complex than that. Indeed BH's emphasis on the limits of theories resembles in some ways Kant's view that the Ding-an-Sich is unknowable. However, we will see below that $\mathrm{BH}$ assume in a realist fashion that there is a sense in which limited domains of mind-independent reality can be known. In this sense they differ from Kant and connect with critical scientific realism (e.g. Niiniluoto, 1999). Their focus on ontology also connects them with the ontological concerns of Heidegger and Merleau-Ponty.

It is clear from the above that $\mathrm{BH}$ do not expect that physics will lead to a "Theory of Everything". Rather, they propose that “... nature in its total reality is unlimited, not merely quantitatively, but also qualitatively in its depth and subtlety of laws and processes" (BH 1993: 321). Indeed, Bohm put forward such a notion of "qualitative infinity of nature" already in his 1957 book Causality and Chance in Modern Physics.

$\mathrm{BH}$ then note that if nature is qualitatively infinite in the way suggested above, it makes it possible that what we at any given time consider reality may always turn out to be "mere appearance" in relation to some more fundamental "essence" which scientific research may discover. However, such discoveries may require speculative new concepts which go far beyond what can be observed at the time of their proposal. This emphasis on the value of concepts about the currently unobservable domain again connects $\mathrm{BH}$ strongly with the realist tradition in the philosophy of science. A key role that concepts about currently unobservable domains or levels can have is that they help us to detect evidence which otherwise might not be recognized at all. For example, the atomic hypothesis (going back to Democritus) made people alert to the kind of large-scale evidence that could support the existence of atoms. By making use of such evidence the initially unobserved atoms have become part of the observable domain (BH 1993: 194).

$\mathrm{BH}$ emphasize that deeper explanations often imply the limited validity of what were previously the basic concepts, while the latter are recovered only as approximations or limiting cases (BH 1993: 322). They point out that this relationship of concepts prevails not merely in physics but is typical of the whole of our experience. As an example they consider what takes place as we go round a circular table: "What we see immediately is an ever-changing elliptical shape. We have learned to regard this changing shape as a mere appearance. The essence (i.e. the true being) is considered to be a rigid circular object." (BH 1993: 322) However, further investigation shows that the table is an atomic structure. The rigid circular object is now considered as appearance, while the essence is the set of constituting atoms. But yet deeper studies show that even the atom is an appearance, while the essence is a nucleus surrounded by electrons. And even these nucleonic particles were seen to be appearances, while the essence was a set of yet more fundamental particles such as quarks, gluons, preons or else sets of excitations of strings (BH 1993: 322).

The ontological lesson is that “... matter is turning more and more into empty space with an ever more tenuous structure of moving elements" (BH 1993: 322). This is even more so in quantum field theory which treats particles as quantised states of a field that extends over the whole space.

$\mathrm{BH}$ then draw attention to the fact that in this historical development something has been constant. There is a pattern in which at each stage certain features are regarded as appearance while other features are regarded as of an essence which explains the appearance on a qualitatively different basis. What is taken as essence at any stage, is later seen to be appearance of a still more fundamental essence (BH 1993: 322). This means that ultimately everything plays both the role of appearance and that of essence, as depicted in Fig. 1.

BH suggest that this pattern never comes to an end. They take this to imply that ultimately all of our thought, including our theories, can be regarded as appearance, not to the senses, but to the mind. They add that what science is aiming for is that these appearances be correct. Correctness here means that the actions flowing from them, such as experiments, be coherent with what the appearances would imply. Incorrect appearances are thus "either mistaken or illusory".

They illustrate this idea with the example of the table. To have the appearance of an elliptical object is correct only in a very limited sense. If we bring in the thought that the object is a rigid circle we obtain a more nearly correct overall set of appearances, while the thought of the atomic structure makes the whole set still more nearly correct and so on.

The example illustrates how we are constantly extending the appearances with the aid of thought. The ultimate reality is "unlimited and unknown" - it is somewhat like Kant's das Ding an Sich. However, "its successive appearances serve as an ever more accurate guide to coherent action in relation to this reality" (BH 1993: 323). One might wonder how this compares with the idea of approaching the final truth. $\mathrm{BH}$ emphasize - in a somewhat phenomenological vein - that our theories are not primarily forms of knowledge about the world but rather, they are forms of insight that arise in our attempts to obtain a perception of a deeper nature of reality as a whole. They add that we should not expect the development of theories ever to come to an end any more than we would look forward to a final sense perception (BH 1993: 323).

\section{Ontology vs. appearance}

We have seen above that BH suggest that even our basic concepts and categories of thought are ultimately appearances. This also applies to the basic concepts of their own ontological interpretation. So, we might ask, are they doing "quantum ontology" or "quantum phenomenology"? Are they reasoning about the beings or about the way the beings appear? Specifically we could ask, if physics essentially involves reasoning about the way the beings appear, what is the point of making an ontological interpretation of

$$
\begin{aligned}
& \text { A1 } \\
& \begin{aligned}
\text { E1 - } 2 \text { A2 }->\text { A3 } \\
\quad \text { E3 }->\text { A4 } 4
\end{aligned}
\end{aligned}
$$

Fig. 1. The pattern of appearances and essences. 
quantum theory? This is, roughly, the question I put to David Bohm in London in the spring of 1991, in the course of the many philosophical discussions I had with him while working on my $\mathrm{PhD}$ thesis. The Undivided Universe was then a manuscript and I had read and commented the draft of ch 14.2. As far as I can remember, Bohm's reply to this question was added to the manuscript and can be found in pp. 324-5 of the book. It seemed to me that Bohm's strong focus on "appearances" and "theories as perception" leaned toward an empiricist, pragmatist, anti-ontological or anti-realist interpretation of science (rather than realist or ontological). This, of course, seemed to be in contradiction with the very name of BH's interpretation ("ontological interpretation"), included in the subtitle of their book.

In their answer to this type of criticism BH (1993: 324-5) point out that every theory is not only a mere appearance. It is more: its basic concepts must be said in some sense to reflect reality within its own domain. How does this differ from other ontological theories? In other ontological theories in physics it is either implied or asserted that such basic concepts correspond to independently existing realities, for example, not dependent on context or deeper levels of being. In BH's approach the basic concepts of a given theory may reflect a reality that is inherently dependent either on context or on deeper levels or on both.

BH thus emphasize that "ontology" is "reasoning about dependent beings", not reasoning about substances (in the sense of ontological independence). Their view constitutes a kind of monism: there is only one independent being, the unlimited and unknowable totality.

To understand this better, let us follow them in reconsidering the example of the table (BH 1993: 324). In what sense are the elliptical appearances of a circular object "realities"? Consider the light coming from the object to the eye and how the eye makes an elliptical image, carried into the brain. This is an essential part of the reality corresponding to the elliptical appearance. This reality is not independently existent. It depends on a context including the above process, especially on the circular object which is the essential meaning of the elliptical appearances. But then even the circular object is found to be dependent for its existence on a wide range of contextual parameters, e.g. temperature and especially the atomic constituents etc. as we go through a series of deeper essences and appearances (BH 1993: 325).

\section{Appearance and essence in the quantum domain}

What about the quantum domain? Remember our question: are $\mathrm{BH}$ reasoning about the beings or about the way the beings appear? How would they answer this question in the quantum domain? They say, interestingly, that in the quantum domain it is no longer possible, even in their ontological approach, to move from appearance to the essence in the way we have described above. Why not? They emphasize that the "beings" in a typical quantum experiment participate irreducibly in each other. This means that it is not possible to locate (individuate) the essence that is supposed to underlie a given appearance.

Consider measuring the momentum of a particle in classical physics. The "appearances" show themselves in the measuring instrument. The "essence" can be thought of as the property (momentum) possessed by the particle. The measuring instrument then typically reveals the momentum that the particle had before the measurement. There is, of course, always a disturbance involved, but this can be accounted for in principle without limit. However, it does not work like this in the quantum domain, where the observed system and the measuring instrument participate irreducibly in each other. $\mathrm{BH}$ emphasize that the appearance (measured value) cannot typically be traced into an underlying essence (state of the observed system before the measurement). Instead, we have to consider appearance and essence (system + apparatus) as a totality ( $\mathrm{BH} 1993$ : 325). The appearances show themselves as large-scale results that are observable in the apparatus, while the essence is the whole quantum ontology of quantum fields and particles, including those of the apparatus and the observed system. The observed momentum typically reflects the state in which the observed system was left after the measurement process is over. In this sense the observed momentum reflects the whole experimental context; and it seems that "undivided wholeness" is lurking behind the appearances at the quantum level. This is very reminiscent of Bohr's "indivisible quantum phenomenon". But we can now understand how this indivisibility is a result of the underlying holistic quantum ontology.

\section{Does Bohm and Hiley's ontological interpretation constitute a form of scientific realism?}

Let us summarize Bohm and Hiley's view about appearance and essence. They say that each form of thought of the essence is an appearance which however also reflects a reality. They emphasize that this reality is always dependent for its existence as well as for its qualities and properties on broader contexts and deeper levels. This is one of the senses in which the theory is ontological, in spite of the fact that all thought gives appearances. Also, BH's approach provides a reflection of reality that is in the form of a totality, including the measuring instruments along with everything else. This implies that BH's approach is a kind of endophysics in the sense that it is not possible to eliminate the effect of the measuring apparatus in, say, momentum measurements.

Is BH's approach realism? Note that in their theory observations of position really disclose position (Bohm and Hiley, 1993: 109-10). The idea of measurement as being able to disclose pre-existing properties seems to be characteristic of realism in the traditional sense. But this is not the case for momentum, as we have emphasized. Typically, the momentum that is measured is created in the act of measurement which means that pre-existing momentum values are not disclosed by the act of momentum measurement. As Arthur Fine (1996) has emphasized, with respect to all other variables than position the Bohm theory is not realist in such traditional sense.

We have also seen how $\mathrm{BH}$ emphasize that the reality that our theories can reflect is always a dependent reality. They assume that the reality that science is dealing with at any stage is inherently dependent either on context (e.g. temperature) or on deeper levels (e.g. atomic structure) or on both. At the quantum level there is the special feature that the object and observing apparatus participate irreducibly in each other. This dependence has major philosophical implications for them.

So, does BH's interpretation constitute a form of scientific realism? We have seen that it is realism in the sense that the independent reality of the universe is assumed, as well as the possibility to perceive the deeper nature of reality as a whole with our theories. However, many features of traditional realism are denied. Theories are primary forms of insight (perceptions, "appearances to the mind"), they are not primarily forms of knowledge about the world. Truth is not primarily correspondence between theories and reality, even if a correct theory "reflects" reality within its own domain. An important aim of science is to develop "correct" theories (appearances to the mind). "Correctedness" is evaluated not in terms of correspondence between propositions and states of affairs but in terms of pragmatic criteria. For according to $\mathrm{BH}$ 
correctedness of a theory requires that the actions flowing from it, such as experiments, be coherent with what the theory implies (cf. Bohm and Pylkkänen, 1991). And the assumption that reality is infinite and unknown implies that science cannot reach a final "Theory of Everything".

Paraphrasing Kant, Arthur Fine (1996) has suggested that "reality without disclosure is blind". BH assume that we can never disclose the whole of the ultimate reality, because it is unlimited and unknown. Is such a BH-style reality without disclosure blind? One thing to note here is that no absolute limits to disclosure are assumed, so the veil is always in principle removable. Also, when BH's quantum ontology is situated into the broader implicate order framework, with the introduction of higher levels of organization, the way is opened to understanding creativity and biological evolution in a new way (Bohm, 1980 ch 7; Bohm and Hiley, 1993; ch 15; Bohm and Peat, 2002; cf. Kauffman and Gare, 2015; for a further discussion of analogies between thought and quantum processes, see Pylkkänen, 2014).

\section{Concluding reflections}

We started off by considering the relationship between phenomenology and the revolutionary philosophical implications of quantum and relativity physics. We then noted how Niels Bohr's interpretation of quantum theory implies the need for a certain kind of epoche or suspension of assumptions about reality at the quantum level. We further saw how even in the context of Bohm and Hiley's ontological interpretation of quantum theory it is not straightforward to adopt the "natural attitude" toward the empirical phenomena. For example, a momentum measurement cannot be understood as a revelation of a pre-existing well-defined property of the observed system. Thus even the Bohm-Hiley interpretation implies the need for a kind of epoché. We have to suspend our natural classical tendency to assume that from the observable large-scale results of measurements we can infer the pre-existing state of the observed system.

It is not clear how literally we should take the analogy between Husserl's epoché and the varieties of quantum epoché we have briefly sketched above. In Husserl's case an important aim of the epoché or "bracketing" is to enable the unbiased study of conscious experience. In Bohr's case suspending assumptions of reality at the quantum level is a crucial part of his subtle notion of complementarity, which captures the mutual exclusivity of certain experimental quantum phenomena. In Bohm and Hiley's case the need to suspend the move from appearance to essence in the context of, say, momentum measurements is a result of the radically participatory nature of quantum ontology. While not denying that these cases are different, it seems fair to say that in all of them the epoche serves the achievement of a clearer and more truthful picture of the domain under interest.

\section{References}

Bacciagaluppi, Valentini, 2009. Quantum Theory at a Crossroads: Reconsidering the 1927 Solvay Conference. Cambridge University Press, Cambridge.

Backman, J., 2010. Heidegger ja fenomenologian asia ([Heidegger and the matter of phenomenology]). In: Miettinen, T., Pulkkinen, S., Taipale, J. (Eds.), Fenomenologian Ydinkysymyksiä [The Core Questions of Phenomenology]. Gaudeamus, Helsinki (Titles and excerpts translated from Finnish by Paavo Pylkkänen).

Bohm, D., 1980. Wholeness and the Implicate Order. Routledge, London.

Bohm, D., 1987. Hidden variables and the implicate order. In: Hiley, B.J., Peat, D.J. (Eds.), Quantum Implications: Essays in Honour of David Bohm. Routledge, London.

Bohm, D., 1990. A new theory of the relationship of mind and matter. Philos. Psychol. 3, 271-286.

Bohm, D., Hiley, B.J., 1975. On the intuitive understanding of nonlocality as implied by quantum theory. Found. Phys. 5 (1), 93-109.

Bohm, D., Hiley, B.J., 1987. An ontological basis for quantum theory: I. Non- relativistic particle systems. Phys. Rep. 144 (6), 323-348.

Bohm, D.. Hiley, B.J., 1993. The Undivided Universe: An Ontological Interpretation of Quantum Theory. Routledge, London.

Bohm, D., Peat, F.D., 2002. Science, Order and Creativity, second ed. Routledge London.

Bohm, D., Pylkkänen, P., 1991. Cognition as a Movement toward Coherence. Unpublished manuscript. The David Bohm papers. Birkbeck Library, University of London.

Bohm, D., Vigier, J.-P., 1954. Model of the causal interpretation of quantum theory in terms of a fluid with irregular fluctuations. Phys. Rev. 96 (1), 208-216.

Bohr N., Discussions with Einstein on Epistemological Problems in Atomic Physics, In: Schilpp P.A. (Ed), 1949

Carson, C., 2011. Modern or antimodern science? Weimar culture, natural science, and the Heidegger-Heisenberg exchange. In: Carson, Cathryn, Kojevnikov, Alexei, Trischler, Helmuth (Eds.), Weimar Culture and Quantum Mechanics: Selected Papers by Paul Forman and Contemporary Perspectives on the Forman Thesis. Imperial College Press, London, pp. 523-542.

Einstein A., Remarks Concerning the Essays Brought Together in this Co-operative Volume, In: Schilpp, P.A. (Ed), 1949

Fine, A., 1996. On the interpretation of Bohmian mechanics. In: Cushing, J.T. Fine, A Goldstein, S. (Eds.), (1996): Bohmian Mechanics and Quantum Theory: an Appraisal, Boston Studies in the Philosophy of Science, vol. 184. Kluwer, Boston.

Flack, R., Hiley, B.J., 2015. Weak measurements, the energy-momentum tensor and the Bohm approach. In: Gao, S. (Ed.), Protective Measurement and Quantum Reality towards a New Understanding of Quantum Mechanics. Cambridge University Press, Cambridge.

Globus, G., 2003. Quantum Closures and Disclosures. John Benjamins, Amsterdam and Philadelphia.

Globus, G., Pribram, K., Vitiello, G. (Eds.), 2004. Being and Brain. At the Boundary between Science, Philosophy, Language and Arts. Advances in Consciousness Research, vol. 58. John Benjamins, Amsterdam.

Goldstein, Sheldon, 2013. "Bohmian Mechanics”, The Stanford Encyclopedia of Philosophy. Spring 2013 Edition. In: Zalta, Edward N. (Ed.). URL. http://plato. stanford.edu/archives/spr2013/entries/qm-bohm/.

Hiley, B.J., Pylkkänen, P., 2005. Can mind affect matter via active information? Mind Matter 3 (2), 7-26. URL. http://www.mindmatter.de/mmpdf/hileywww.pdf.

Holland, P., 2011. A quantum of history. Contemp. Phys. 52, 355.

Honner, J., 1987. The Description of Nature: Niels Bohr and the Philosophy of Quantum Physics. Oxford University Press, Oxford.

Husserl, E., 1913/1976. Ideas. General Introduction to Pure Phenomenology. Translated by W.R. Royce Gibson. George Allen and Unwin, London.

Hut, P., 2001. The Role of Husserl's Epoche for Science: a View from a Physicist invited paper presented at the 31st Husserl Circle conference in Bloomington IN, in February 2001. URL. https://www.ids.ias.edu/ piet/publ/other| husserlcircle.html.

Kauffman, S., Gare, A., 2015. Beyond Descartes and Newton: Recovering life and humanity. In: Simeonov, P.L., Rosen, S.M., Gare, A. (Eds.), Special Theme Issue on Integral Biomathics: Life Sciences, Mathematics, and Phenomenological Philosophy, Prog. Biophysics Mol. Biol., vol. 19. Issue 2.

Marshall, C., 2012. Appearance and Reality, in New World Encyclopedia Permanent URL. http://www.newworldencyclopedia.org/p/index.php?title=Appearance and Reality\&oldid $=964607$.

Merleau-Ponty, M., 2003. In: Seglard, D. (Ed.), Nature: Course Notes from the College de France. Northwestern. R Vallier, trans.

Ney, A., 2013. Introduction, in Ney, A., and Albert, D., (eds).

Ney, A., Albert, D. (Eds.), 2013. The Wave Function: Essays on the Metaphysics of Quantum Mechanics. Oxford University Press.

Niiniluoto, I., 1999. Critical Scientific Realism. Oxford University Press, Oxford.

Plotnitsky, A., 1994. Complementarity. Anti-epistemology after Bohr and Derrida. Duke University Press, Durham and London.

Plotnitsky, A., 2010. Epistemology and probability. Bohr, Heisenberg, Schrödinger and the Nature of Quantum-theoretical Thinking. Springer, Heidelberg and New York.

Pylkkänen, P., 2007. Mind, Matter and the Implicate Order. Springer Frontiers Collection, Heidelberg and New York.

Pylkkänen, P., 2014. Can quantum analogies help us to understand the process of thought? Mind Matter 12 (1), 61-92. URL. http://www.mindmatter.de/ resources/pdf/pylkkaenen_www.pdf.

Pylkkänen, P., 2015a. Fundamental physics and the mind - is there a connection? In: Atmanspacher, H., et al. (Eds.), Quantum Interaction. 8th International Conference. Springer, Berlin, pp. 3-11.

Pylkkänen, P., 2015b. Is there room in quantum ontology for a genuine causal role of consciousness? In: Khrennikov, A., Haven, Emmanuel (Eds.), The Palgrave Handbook of Quantum Models in Social Science. Palgrave Macmillan (forthcoming).

Pylkkänen, P., Hiley, B.J., Pättiniemi, I., December 2015. Bohm's approach and individuality. To appear. In: Guay, A., Pradeu, T. (Eds.), Individuals across Sciences: A Revisionary Metaphysics? Oxford University Press, Oxford (estimated). http:/ arxiv.org/abs/1405.4772.

Pylkkö, P., 1998. The Aconceptual Mind: Heideggerian Themes in Holistic Naturalism. John Benjamins, Amsterdam and Philadelphia.

Saunders, S., et al. (Eds.), 2010. Many Worlds? Everett, Quantum Theory, \& Reality. Oxford University Press.

Schilpp, P.A., 1949. Albert Einstein: Philosopher-scientist. In: The Library of Living Philosophers, vol. 7. The Library of Living Philosophers, Evanston, IL 
Towler, M., 2009. Pilot-wave Theory, Bohmian Metaphysics, and the Foundations of Quantum Mechanics, a Graduate Course at the Cavendish Laboratory. University of Cambridge. http://www.tcm.phy.cam.ac.uk/ mdt26/pilot waves.html.
Westerlund, F., 2014. Heidegger and the Problem of Phenomenality. In: Philosophical Studies from the University, vol. 45. Unigrafia, Helsinki. URL. https:/ helda.helsinki.fi/bitstream/handle/10138/45258/Westerlund_vaitoskirja.pdf? sequence $=1$ 